\title{
Cutaneous leukocytoclastic vasculitis: the role of lymphocytes and related immune markers
}

\author{
Thilo Gambichler, Magdalena A. Kulik, Marina Skrygan, Isabelle Rooms, Stefan Höxtermann
}

Department of Dermatology, Ruhr-University Bochum, Bochum, Germany

Adv Dermatol Allergol 2017; XXXIV (4): 299-305

DOI: https://doi.org/10.5114/ada.2017.69307

\begin{abstract}
Introduction: Apart from neutrophils, other immune cells may play a significant pathogenetic role in cutaneous leukocytoclastic vasculitis (CLV).

Aim: To investigate lymphocytes and related immunological factors in patients with CLV requiring systemic glucocorticosteroid treatment.

Material and methods: Fourteen patients with severe idiopathic CLV were treated with systemic prednisolone in a tapered dose regimen. Ten healthy individuals served as controls. At baseline and post-treatment, we studied inducer/helper and suppressor/cytotoxic T lymphocytes, B lymphocytes, natural killer cells, CD4+CD25++CD127- cells, CD4+CD25+CD39+ cells and FOXP3, transforming growth factor $\beta 1$ (TGF- $\beta 1$ ) and interleukin-10 (IL-10) mRNA levels in the blood using flow cytometry and real time polymerase chain reaction (RT-PCR), respectively. On immunohistochemistry, we studied CD4, CD8, granzyme B, TGF- $\beta 1$, and IL-10.

Results: Flow cytometry did not show significant differences. The RT-PCR revealed that TGF- $\beta 1 \mathrm{mRNA}$ expression was significantly higher after therapy when compared to baseline and controls. On immunohistology, baseline CLV lesions showed significantly more CD4+ lymphocytes than post-treated CLV and controls. CD8+ expression was significantly higher after therapy when compared to baseline and controls. Baseline granzyme B was significantly increased when compared to treated CLV and controls. The IL-10 expression of treated CLV was significantly increased when compared to baseline CLV and; baseline CLV IL-10 expression was significantly increased as compared to controls.

Conclusions: Circulating T regulatory cells do not play a significant role in the pathogenesis of CLV. T helper cells and granzyme B seem to be involved in the inflammatory cutaneous process of CLV. A resolution of CLV observed after glucocorticosteroid treatment may be mediated via up-regulation of TGF- $\beta 1$ and IL-10 in different compartments.
\end{abstract}

Key words: cutaneous vasculitis, lymphocytes, cytokines.

\section{Introduction}

Cutaneous leukocytoclastic vasculitis (CLV), also known as hypersensitivity vasculitis and hypersensitivity angiitis, is a histopathologic term commonly used to denote a small-vessel vasculitis. The CLV is usually not associated with systemic vasculitis or glomerulonephritis etc. [1]. The main clinical feature of CLV is palpable purpura, primarily affecting dependent areas such as legs, ankles, feet and buttocks. Based on current data, CLV is associated with the following conditions: idiopathic (45-55\%), infection (15-20\%), inflammatory disease (15-20\%), drug intake (10-15\%), and malignancies (5\%) [2]. Acute stage CLV is characterized by a predominate infiltrate of neutrophils in and around the vessel wall and the lumen, but without evidence of new capillary formation and fibrous proliferation in and around the vessel. Another feature is that the internal elastic lamina remains intact. In the subacute stage, CLV is characterized by a mixed infiltrate of neutrophils, lymphocytes and histiocytes with the formation of a fibrinoid deposit (i.e., fibrinoid necrosis) in the intima of the affected arteries. The subacute stage is then followed by a reparative (i.e., granulation tissue) and healed stage (i.e., scaring) [1]. Usually, vascular immunoglobulin (IgM, IgG) and complement (C3) deposits are found by direct immunofluorescence assessments implicating immune complex deposition in the pathogenesis of CLV. However, the exact pathomechanism of CLV is obscure [2].

So far, there have been no systematic studies investigating T cell subsets and other immune cells in CLV. In particular, T regulatory cells (Tregs) are currently of great interest in the pathogenesis of many human diseases, particularly such as autoimmune disorders. Tregs are now recognized as the mediators of peripheral tolerance and potent suppressors of excessive immune responses. Several Treg subtypes with distinct phenotypes, cytokine production

Address for correspondence: Prof. Dr. Thilo Gambichler, Department of Dermatology, Ruhr-University Bochum, Gudrunstrasse 56, 44791 Bochum, Germany, phone: +49-234-509-3459, fax: +49-234-509-3445, e-mail: t.gambichler@klinikum-bochum.de Received: 1.06.2017, accepted: 20.06.2017. 
profiles, and modes of action have been described $[3,4]$. In the CD4+ regulatory $T$ cell compartment, $C D 4^{+} \mathrm{CD} 25^{+}$ $T$ cells and interleukin 10-producing type $1 \mathrm{~T}$ regulatory cells have been described. Other markers for Tregs include CD4+CD25++CD127- and CD4+CD25+CD39 [3, 4].

\section{Aim}

Hence, we aimed to focus on the assessment and comparison of lymphocytes - in particular Tregs - and related immune markers in patients with CLV and controls who underwent treatment with systemic prednisolone.

\section{Material and methods}

\section{Subjects}

Fourteen patients (10 males, 4 females; mean \pm SD age: $42.6 \pm 13.7$ years) with severe (ulceration, skin necrosis etc.) and/or recurrent flares of CLV were enrolled in the study after giving informed consent. The diagnosis of CLV was verified by patient's history, clinical symptoms, histological findings, and laboratory studies [1, 2]. Systemic manifestations of vasculitis were excluded by means of laboratory tests (e.g. urine analysis, serum creatinine, ANA, ANCA, cryoglobulins) and abdomen ultrasonography. In all patients, direct immunofluorescence studies were performed. Patients were treated with an initial dose of intravenous prednisolone $(1.5 \mathrm{mg} / \mathrm{kg}$ body weight) which was slowly tapered. Treatment outcome was estimated using a simple score: $0=$ none improvement/worsening; 1 = slight improvement; 2 = moderate improvement; 3 = good to excellent improvement. Ad ditionally, we included 10 healthy sex/age-matched controls (age: $45.8 \pm 15.3$ years). The study was approved by the ethics review board of the Ruhr-University Bochum (\#4088-11) and conducted according to the Declaration of Helsinki principles.

\section{Flow cytometry}

In order to identify and determine the absolute counts of lymphocytes in the peripheral blood, flow cytometric methods were applied using antibodies for $T$ lymphocytes (CD3+), B lymphocytes (CD19+), helper/inducer T lymphocytes (CD3+CD4+), suppressor/cytotoxic T lymphocytes (CD3+CD8+), natural killer (NK) cells (CD3-CD16+CD56+), and Tregs (CD4+CD25++CD127-, CD4+CD25+CD39+). The FACSCanto II ${ }^{T M}$ cytometer (BD Biosciences, San Jose, CA, USA), equipped with $633 \mathrm{~nm}$ and $488 \mathrm{~nm}$ red and blue lasers, together with computer hardware and FACSDivasoftware ${ }^{T M}$ were used to analyze the aforementioned lymphocyte populations and subsets.

\section{Real-time polymerase chain reaction (RT-PCR)}

Quantitative analysis of real-time RT-PCR was performed in duplicate as previously suggested [5]; total cellular RNA was isolated from blood samples of CLV patients and controls. The quantities of FOXP3, transforming growth factor $\beta 1$ (TGF- $\beta 1$ ) and interleukin-10 (IL-10) mRNA levels were determined using the median log transform of the gene expression. Relative mRNA expression levels were calculated using the comparative $\Delta-\Delta \mathrm{C}_{t}$ method as previously suggested by Livak and Schmittgen [5]. Quantities of all targets in the test samples were normalized to the corresponding RPL38 RNA transcript in the blood samples.

\section{Routine histology, direct immunofluorescence and immunohistochemistry}

Four millimetre punch biopsies were performed in lesional skin of patients (at baseline and after 7 days) and healthy skin of controls. Biopsy sites were the lower legs. In order to confirm clinical diagnosis, routine histology (haematoxylin-eosin staining) and direct immunofluorescence were performed in all CLV patients. For immunohistochemistry, paraffin-embedded sections were mounted on silanized slides and stored for $1 \mathrm{~h}$ in a humid chamber at $60^{\circ} \mathrm{C}$. Sections were deparaffinized in xylene and washed with 100\%, 96\%, 70\% and 50\% ethanol for 5 min each and rinsed with demineralized water. Immunohistochemical staining was eventually performed for CD4 and CD8, granzyme B, transforming growth factor- $\beta 1$ (TGF- $\beta 1$, Abcam, Cambridge, UK), and interleukin 10 (IL-10, Antibodies-online, Atlanta, USA). Immunohistochemistry specimens were analysed using a Zeiss (Jena, Germany) light microscope at 400× magnification in 5 fields of view. Quantitative results were expressed as the relative proportion of positive dermal infiltrating cells $(0=100 \%)$. The CD4/CD8 ratio was calculated from the mean percentage of positive cells for each case. Mean values of immunoreactivity scores were used for statistical analysis.

\section{Statistical analysis}

Data analysis was performed using the statistical package MedCalc Software (Mariakerke, Belgium). Distribution of data was assessed by means of the D'AgostinoPearson test. Based on distribution data were expressed as medians (range) or means \pm SD. The results were analyzed using the Mann-Whitney test, Wilcoxon test, and paired and un-paired $t$-test. Correlation analysis was performed using the Pearson and Spearman procedure. This was an explorative pilot study without sample size calculation and adjustment for multiple testing. Differences were considered significant when $p<0.05$.

\section{Results}

\section{Clinical data}

In all patients, the diagnosis of CLV was confirmed by histology and direct immunofluorescence. In-patient treatment was performed for median 7 days (5-13 days). 
After treatment clinical improvement was estimated by one observer (M.K.) as follows: 1 (8.3\%) patient had slight improvement, 2 (16.7\%) patients had moderate and 9 (75\%) showed good to excellent improvement. Baseline C-reactive protein levels (28.4 mg/l (5.6-69.4 mg/l)) were significantly ( $p=0.039$ ) increased when compared to post-

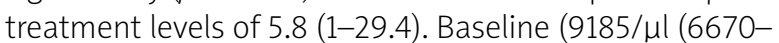
$12570 / \mu \mathrm{l}))$ and post-treatment (10850/ $\mathrm{\mu l}(8190-14250 / \mu \mathrm{l}))$ blood leukocyte levels did not significantly $(p=0.084)$ differ, although there was a trend for higher post-treatment leukocytes (likely because of prednisolone intake).

\section{Flow cytometry}

As shown in Table 1 in detail, flow cytometry for CD4+, CD8+, CD4+CD25++CD127-, or CD4+CD25+CD39+ T lymphocytes, B lymphocytes and natural killer cells did not show significant differences between the groups assessed.

\section{Real time polymerase chain reaction}

RT-PCR revealed that TGF- $\beta 1$ mRNA expression was significantly higher after therapy $(13.3 \pm 8.1)$ when compared to baseline $(6 \pm 4.3, p=0.0065)$ and controls (5.6 $\pm 3.9, p=0.026$ ). mRNA expression of FOXP3 and IL-10 did not significantly differ between the groups (Table 1). Baseline TGF- $\beta 1$ and IL-10 mRNA levels of CLV patients significantly ( $p=0.0051)$ correlated as indicated by a high coefficient of correlation of 0.83 (Figure 1).

\section{Immunohistochemistry}

On immunohistology (Table 2 and Figure 2), baseline CLV lesions (42\% (6.3-70\%)) showed significantly more CD4+ lymphocytes than post-treated CLV $(20.8 \%$ (8.2-37.5\%, $p=0.0068)$ and controls (18.4\% (6.5-26\%)). CD8+ expression was significantly higher after therapy (32.1\% (12-48.9\%)) when compared to baseline (18.3\% (5.6-34.7\%), $p=0.024)$ and controls (16.5\% (8.2-31\%), $p=0.0055)$. After treatment, the CD4/CD8 (0.68\% (0.22$0.94)$ ratio was significantly decreased when compared to baseline CLV (2.6 (0.43-5.7), $p=0.001)$ and controls (1.31 (0.9-1.55), $p=0.0061)$. Baseline granzyme $\mathrm{B}$ expression $(21.9 \%(2-40 \%))$ was significantly increased when compared to treated CLV (0\% (0-8\%), $p=0.0005)$ and controls (0\% (0-4\%), $p=0.0004)$. IL-10 expression of treated CLV (84.4\% (70-91)) was significantly increased when compared to baseline CLV (75.2\% (54.6-84\%), $p=$ $0.0005)$ and controls (14.2\% (8-24\%), $p=0.0004)$; baseline CLV IL-10 expression was also significantly increased as compared to controls ( $p=0.0004)$.

\section{Discussion}

Overexpression of FOXP3 has been shown to lead to a hypoactive immune state suggesting that it is a central regulator of T cell activity. FOXP3 is a more specific marker of Tregs than most cell surface markers such as

Table 1. Flow cytometry and gene expression analysis in the blood of patients with cutaneous leukocytoclastic vasculitis and controls

\begin{tabular}{|c|c|c|c|}
\hline Parameter & $\begin{array}{c}\text { A } \\
\text { Cutaneous leukocytoclastic } \\
\text { vasculitis } \\
\text { Pre-treatment }\end{array}$ & $\begin{array}{c}\text { B } \\
\text { Cutaneous leukocytoclastic } \\
\text { vasculitis } \\
\text { Post-treatment }\end{array}$ & $\begin{array}{c}\text { C } \\
\text { Controls }\end{array}$ \\
\hline \multicolumn{4}{|l|}{ Flow cytometry: } \\
\hline 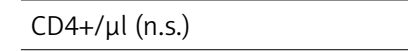 & 799 (123-2980) & $1066(218-2112)$ & $644(433-1329)$ \\
\hline 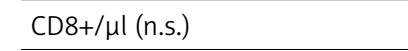 & $274(48-887)$ & $331(75-836)$ & $296(147-631)$ \\
\hline CD4/CD8 ratio (n.s.) & $3(0.56-11.5)$ & $2.9(0.9-15.6)$ & $2.5(0.9-4.4)$ \\
\hline CD4+CD25++CD127- (\%) (n.s.) & $6.4(3.5-17)$ & $7.6(1.3-11.9)$ & $5.7(2.4-8.9)$ \\
\hline CD4+CD25+CD39+ (\%) (n.s.) & $4(0.6-13.1)$ & $3.4(1.7-10.8)$ & $2(0.7-6.2)$ \\
\hline 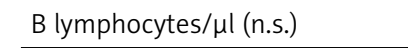 & $190(35-876)$ & $473(67-670)$ & $186(55-577)$ \\
\hline Natural killer cells/ul (n.s.) & $162(59-691)$ & $158(44-827)$ & $183(108-337)$ \\
\hline \multicolumn{4}{|l|}{ Real-time RT-PCR ${ }^{\S}$ : } \\
\hline FOXP3 (n.s.) & $0.067 \pm 0.051$ & $0.045 \pm 0.091$ & $0.099 \pm 0.068$ \\
\hline $\begin{array}{l}\text { Transforming growth factor- } \beta 1^{*} \\
\text { A vs. B, } p=0.0065 \\
\text { B vs. C, } p=0.026\end{array}$ & $6 \pm 4.3$ & $13.3 \pm 8.1$ & $5.6 \pm 3.9$ \\
\hline Interleukin 10 (n.s.) & $0.017 \pm 0.005$ & $0.011 \pm 0.006$ & $0.02 \pm 0.02$ \\
\hline
\end{tabular}




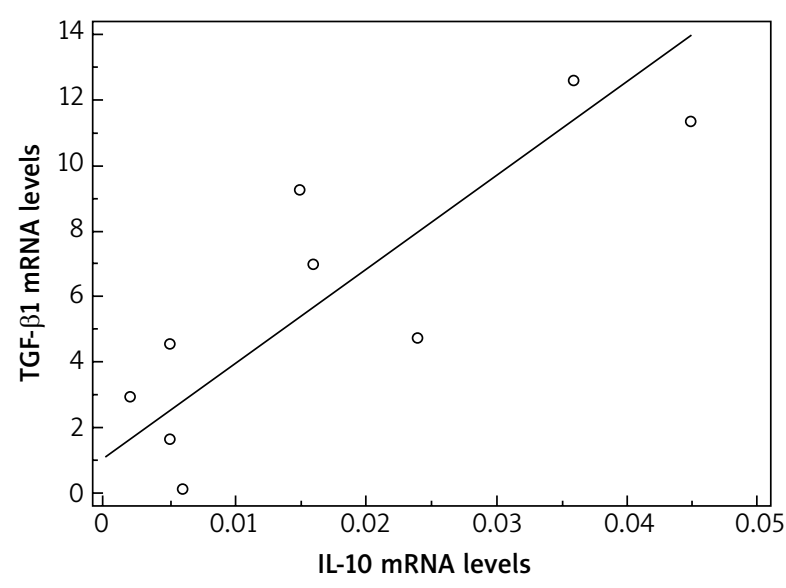

Figure 1. Significant correlation $(r=0.83 ; p=0.0051)$ of circulating TGF- $\beta 1$ and IL-10 mRNA levels in cutaneous leukocytoclastic vasculitis before systemic treatment with prednisolone

CD4 and CD25. By contrast, CD4+CD25++CD127- T cells are the best known membrane markers for Tregs which can be used for natural occurring Treg isolation in human blood. Moreover, CD4+CD25+CD39+ T cells represent activated/memory Tregs [3, 4]. Tregs have relatively rarely been studied in vasculitic disorders. Klapa et al. [6] as well Morgan et al. [7] have shown that patients with granulomatosis with polyangiitis - an ANCA-associated systemic small vessel vasculitis - demonstrate a decrease in the number and function of Tregs in the blood and this is most pronounced in those with most active disease [7]. Olivito et al. [8] reported that the frequencies of CD4+CD25 +FOXP3+ Tregs in the blood were significantly lower during the acute phase of Kawasaki's disease than healthy donors and a similar tendency was revealed for FOXP3 mRNA transcripts. FOXP3 expression increased significantly, at both protein and mRNA levels, after intravenous immunoglobulin therapy treatment [8].

Yang et al. [9] studied patients with Henoch-Schönlein purpura (HSP) which is a small vessel vasculitis closely related to CLV. They found that compared with healthy controls, the proportions of T helper 2 (Th2) cells in patients with HSP were significantly higher, and the ratio of Th1/Th2 was remarkably decreased. The proportions of Tregs in patients with HSP were significantly lower than those of controls. Moreover, Yang et al. [9] observed that the mRNA expression of FOXP3 was significantly decreased in patients with HSP when compared to controls. Sengar et al. [10] have shown that during the active phase, HSP patients developed significant lymphocytosis, whereby the absolute numbers of CD4+ cells were significantly increased but absolute numbers of CD8+ cells were not altered.

Unlike in systemic vasculitides, such as granulomatosis with polyangiitis, CLV does not seem to be associated with abnormalities of circulation Tregs, In the present study, neither flow cytometry nor RT-PCR revealed significant differences in circulating Tregs among the groups compared. Moreover, the number of cytotoxic cells (CD8+), B lymphocytes and natural killer cells did

Table 2. Immunohistochemistry of cutaneous leukocytoclastic vasculitis

\begin{tabular}{|c|c|c|c|}
\hline Parameter & $\begin{array}{c}\text { A } \\
\text { Cutaneous leukocytoclastic } \\
\text { vasculitis } \\
\text { Pre-treatment }\end{array}$ & $\begin{array}{c}\text { B } \\
\text { Cutaneous leukocytoclastic } \\
\text { vasculitis } \\
\text { Post-treatment }\end{array}$ & $\begin{array}{c}\text { C } \\
\text { Controls }\end{array}$ \\
\hline $\begin{array}{l}\text { CD4*: } \\
\text { A vs. } B, p=0.0068 \\
\text { A vs. } C, p=0.0088\end{array}$ & $42 \%(6.3-70 \%)$ & $20.8 \%(8.2-37.5 \%)$ & $18.4 \%(6.5-26 \%)$ \\
\hline $\begin{array}{l}\text { CD8*: } \\
\text { B vs. A, } p=0.024 \\
\text { B vs. C, } p=0.005\end{array}$ & $18.3 \%(5.8-34.7 \%)$ & $32.1 \%(12-48.9 \%)$ & $16.5 \%(8.2-31 \%)$ \\
\hline $\begin{array}{l}\text { CD4/CD8 ratio*: } \\
\text { B vs. A, } p=0.001 \\
\text { B vs. } C, p=0.0061\end{array}$ & $2.6 \%(0.43-5.7 \%)$ & $0.68 \%(0.22-0.94 \%)$ & $1.31 \%(0.9-1.55 \%)$ \\
\hline $\begin{array}{l}\text { Granzyme } B^{*}: \\
\text { A vs. } B, p=0.0005 \\
\text { A vs. } C, p=0.0004\end{array}$ & $21.9 \%(2-40 \%)$ & $0 \%(0-8 \%)$ & $0 \%(0-4 \%)$ \\
\hline $\begin{array}{l}\text { Interleukin } 10^{*}: \\
\text { B vs. A, } p=0.0005 \\
\text { B vs. C, } p=0.0004 \\
\text { A vs. C, } p=0.0004\end{array}$ & $75.2 \%(54.6-84.2 \%)$ & $84.4 \%(70-91 \%)$ & $14.2 \%(8-24 \%)$ \\
\hline Transforming growth factor- $\beta 1$ & $20.9 \%(2.3-80 \%)$ & $12.9 \%(3.5-60.7 \%)$ & $25 \%(4-70 \%)$ \\
\hline
\end{tabular}

Pair-wise comparisons for dependent and independent data (Wilcoxon, Mann-Whitney, paired and unpaired t- tests) did not reveal statistically significant differences, except for *. 
A

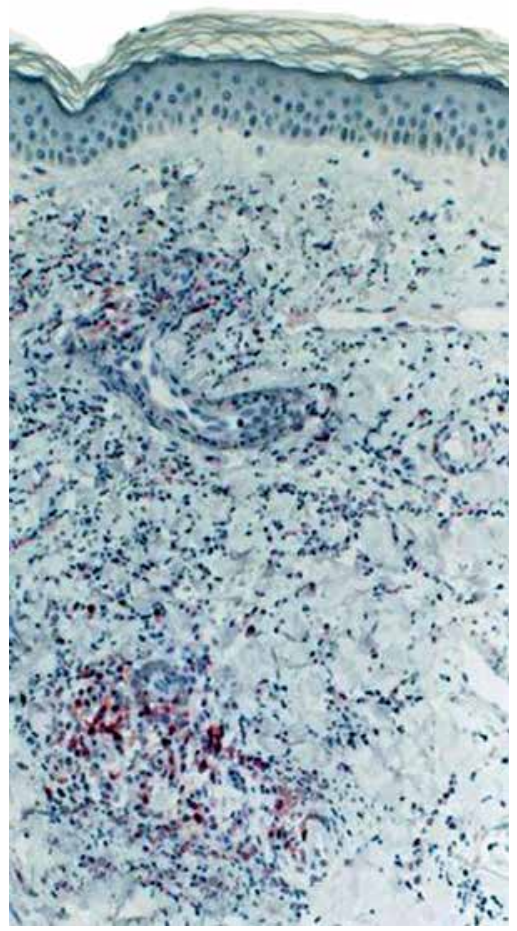

D

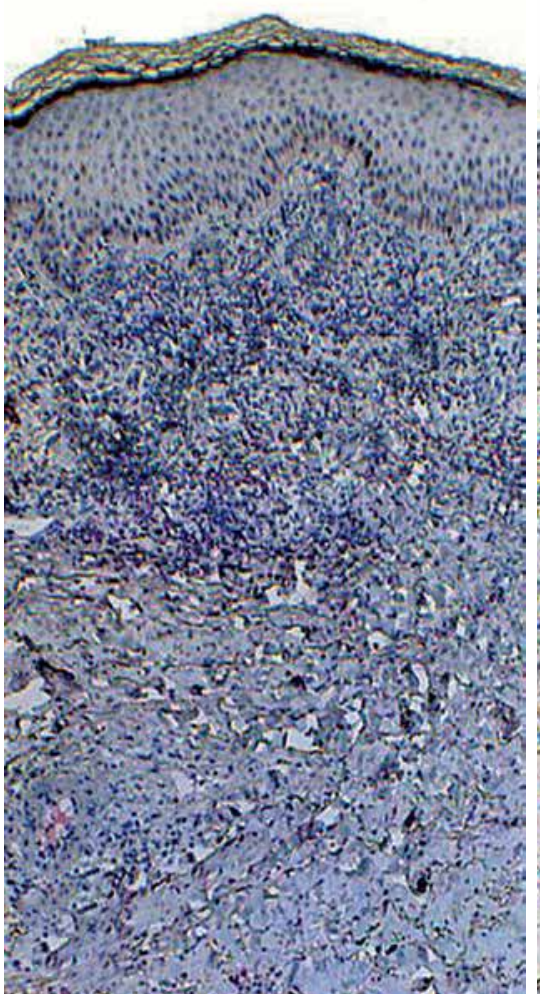

B

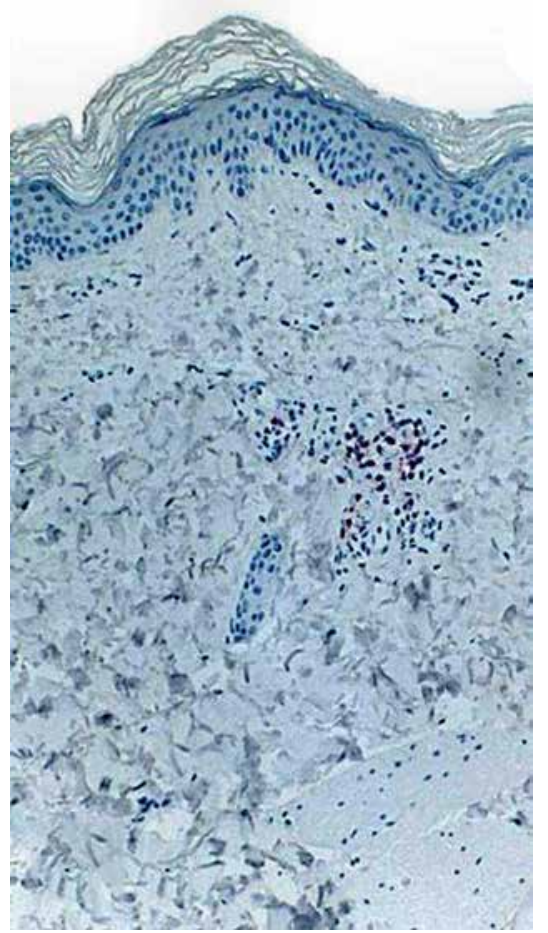

E

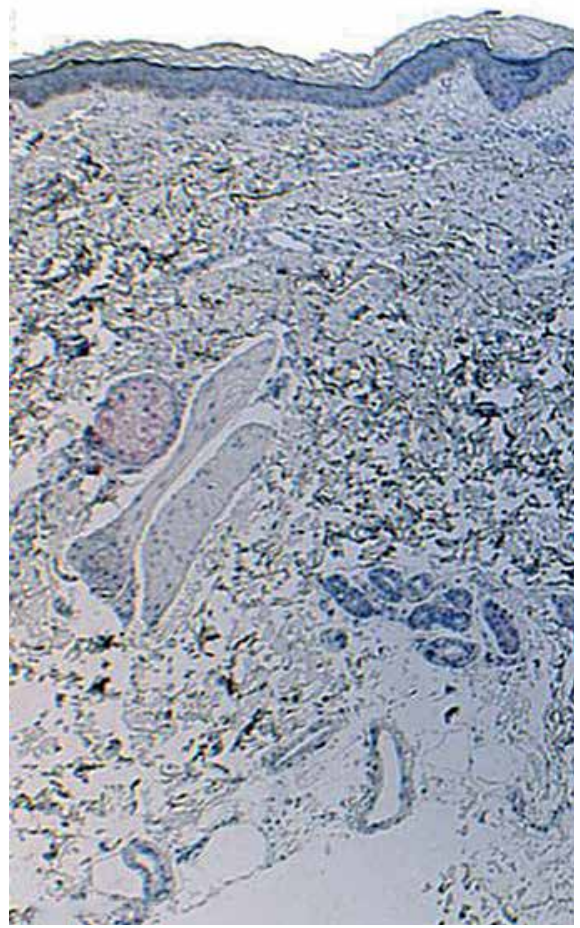

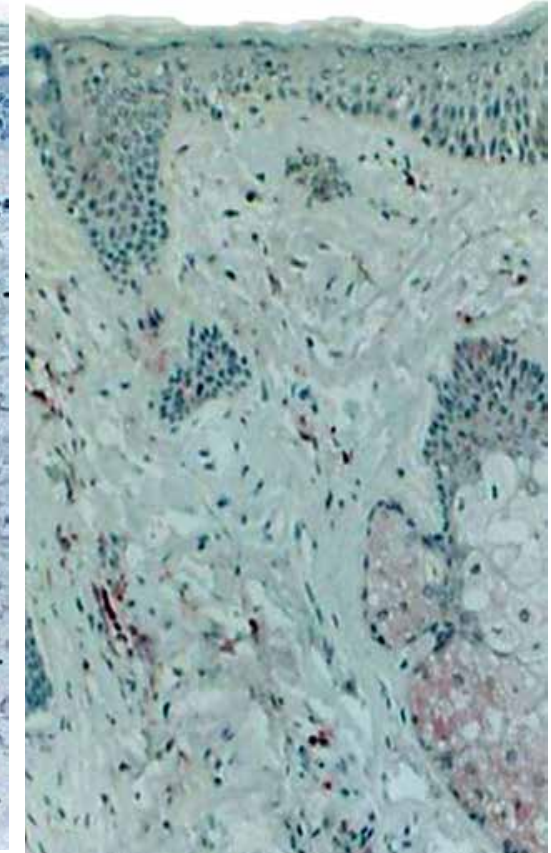

F

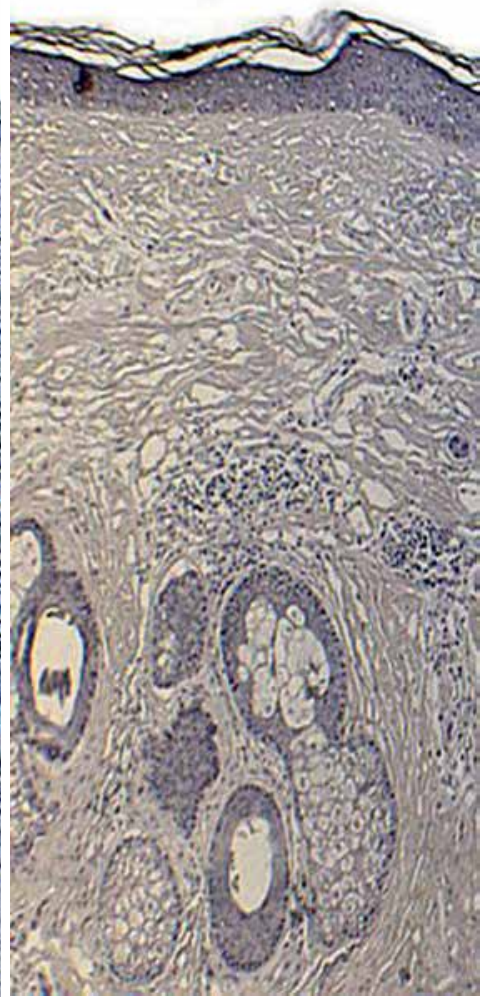

Figure 2. Immunohistopathology image (magnification, 250x) showing increased CD4+ lymphocytes in cutaneous leukocytoclastic vasculitis (A) before treatment when compared to post-treatment skin lesions (B) and healthy controls (C). Granzyme B protein expression is significantly increased in cutaneous leukocytoclastic vasculitis (D) as compared to post-treatment skin lesions (E) and healthy controls (F) 
not significantly differ in baseline blood of CLV patients and controls. The aforementioned data of the present study may support in part the fact that CLV is a condition in which systemic involvement is usually absent [11]. However, we observed that TGF- $\beta 1$ mRNA expression was significantly increased after therapy when compared to baseline and controls. We explain the post-treatment increase in TGF- $\beta 1$ expression in blood with systemic glucocorticosteroid therapy that can induce the expression of TGF- $\beta 1$. Ossege et al. [12] reported that during and after treatment with systemic glucocorticosteroid therapy, the TGF- $\beta 1$ mRNA blood levels significantly increased at day 3-5 and declined about 3 days after discontinuation of therapy. TGF- $\beta 1$ is an immunosuppressant cytokine which blocks cytokine synthesis and hence $T$ cell activation. It has been shown by Huss et al. [13] however that TGF- $\beta$ signaling causes binding of Smad4 to the IL-10 promoter, providing molecular evidence for TGF- $\beta$-mediated IL-10 production from Th1 effector cells. Accordingly, we observed a very strong correlation between TGF- $\beta 1$ and IL-10 mRNA levels in the blood of CLV patients.

It is well known that neutrophils play an important pathogenetic role in the early stage of CLV. Over time, however, lymphocytes are increasingly observed in CLV lesions and may also pathogenetically involved in the condition. With regard to lymphocytic infiltration, our immunohistology data indicate that inflammation in CLV is mainly driven by T helper cells accompanied by increased granzyme B expression. The latter is a pivotal mediator of apoptosis used by cytotoxic T lymphocytes and natural killer cells. The presence of granzyme B in CD4+ T cells was reported by several studies and was believed to be associated with their cytotoxic activity. However, granzyme B can also act upon the cells harboring them [14]. The relatively increased percentage of CD8+ cells after treatment is likely due to the decrease of CD4+ cells. Whether the marked reduction of T helper cells was caused by granzyme B-mediated apoptosis cannot be answered based on the present data. IL-10 was significantly higher expressed in untreated and treated CLV than in the skin of controls. Interestingly, IL-10 mRNA levels were increased even during therapy when compared to baseline. Glucocorticosteroids are known to induce immunotolerance via up-regulation of IL-10 [15]. However, limitations of the present study particularly include a small sample size and lack of functional studies. Moreover, we did not include a CLV control group not receiving systemic steroids. However, this pilot study was not designed to prove the efficacy of systemic prednisolone in CLV. Since CLV represents usually a self-limited, single-episode phenomenon, the treatment should rather consist of general measures such as leg elevation, warming, avoidance of standing, cold temperatures and tight fitting clothing, and therapy with antihistamines, aspirin, or non-steroidal anti-inflammatory drugs [16, 17].
Nevertheless, we studied more severe and/or recalcitrant cases requiring systemic treatment.

\section{Conclusions}

The data of the present study do not indicate a significant role of circulating Tregs in the pathogenesis of CLV. T helper cells and granzyme B seem to be involved in the inflammatory process of CLV. A resolution of CLV after glucocorticosteroid treatment may be mediated via upregulation of TGF- $\beta 1$ and IL-10 in different compartments.

\section{Acknowledgments}

Thilo Gambichler and Magdalena A. Kulik equally contributed to this work.

This work is part of the doctoral thesis of Magdalena Kulik.

\section{Conflict of interest}

The authors declare no conflict of interest.

\section{References}

1. Advances in the Diagnosis and Treatment of Vasculitis. Amezcua-Guerra LM (ed.). InTech Europe, Rijeka, 2011.

2. Fiorentino DF. Cutaneous vasculitis. J Am Acad Dermatol 2003; 48: 311-40.

3. Buckner JH. Mechanisms of impaired regulation by CD4(+) CD25(+)FOXP3(+) regulatory T cells in human autoimmune diseases. Nat Rev Immunol 2010; 10: 849-59.

4. Schuler PJ, Harasymczuk M, Schilling B, et al. Separation of human CD4+CD39+ T cells by magnetic beads reveals two phenotypically and functionally different subsets. I Immunol Methods 2011; 369: 59-68.

5. Livak KJ, Schmittgen TD. Analysis of relative gene expression data using real-time quantitative PCR and the 2(-Delta Delta $C(T)$ ) method. Methods 2001; 25: 402-8.

6. Klapa S, Mueller A, Csernok E, et al. Lower numbers of FoxP3 and CCR4 co-expressing cells in an elevated subpopulation of CD4+CD25high regulatory T cells from Wegener's granulomatosis. Clin Exp Rheumatol 2010; 28 (1 Suppl. 57): 72-80.

7. Morgan MD, Day CJ, Piper KP, et al. Patients with Wegener's granulomatosis demonstrate a relative deficiency and functional impairment of T-regulatory cells. Immunology 2010; 130: 64-73.

8. Olivito B, Taddio A, Simonini G, et al. Defective FOXP3 expression in patients with acute Kawasaki disease and restoration by intravenous immunoglobulin therapy. Clin Exp Rheumatol 2010; 28 (1 Suppl 57): 93-7.

9. Yang J, Li CR, Li YB, et al. The correlation between Kawasaki disease and polymorphisms of tumor necrosis factor alpha and interleukin-10 gene promoter. Zhonghua Er Ke Za Zhi 2003; 41: 598-602.

10. Sengar DP, Acharya CD, Wolfish NM. HLA specificities, lymphocyte subsets, and mitogenic response in Henoch-Schönlein purpura nephritis. Int J Pediatr Nephrol 1984; 5: 197-200.

11. Loricera J, Blanco R, Ortiz-Sanjuán F, et al. Single-organ cutaneous small-vessel vasculitis according to the 2012 revised International Chapel Hill Consensus Conference Nomen- 
clature of Vasculitides: a study of 60 patients from a series of 766 cutaneous vasculitis cases. Rheumatology (Oxford) 2015; 54: 77-82

12. Ossege LM, Sindern E, Voss B, Malin JP. Corticosteroids induce expression of transforming-growth-factor-beta1 mRNA in peripheral blood mononuclear cells of patients with multiple sclerosis. J Neuroimmunol 1998; 84: 1-6.

13. Huss DJ, Winger RC, Cox GM, et al. TGF-beta signaling via Smad4 drives IL-10 production in effector Th1 cells and reduces T-cell trafficking in EAE. Eur I Immunol 2011; 41: 2987-96.

14. Devadas S, Das J, Liu C, et al. Granzyme B is critical for T cell receptor-induced cell death of type 2 helper T cells. Immunity 2006; 25: 237-47.

15. Verhoef CM, van Roon JA, Vianen ME, et al. The immune suppressive effect of dexamethasone in rheumatoid arthritis is accompanied by upregulation of interleukin 10 and by differential changes in interferon gamma and interleukin 4 production. Ann Rheum Dis 1999; 58: 49-54.

16. Micheletti RG, Werth VP. Small vessel vasculitis of the skin. Rheum Dis Clin North Am 2015; 41: 21-32.

17. Carlson JA, Cavaliere LF, Grant-Kels JM. Cutaneous vasculitis: diagnosis and management. Clin Dermatol 2006; 24: 414-29. 